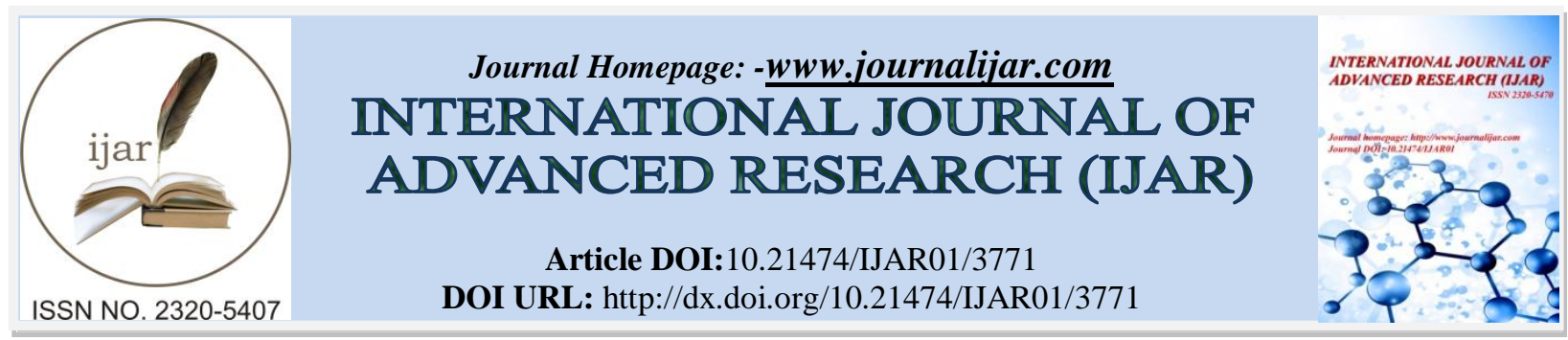

RESEARCH ARTICLE

\title{
A NOTE ON STABILITY ANALYSIS OF COMPARTMENTAL MATHEMATICAL MODEL FOR THE SPREAD OF MALARIA.
}

A. Ali ${ }^{1}$, R. Hussain ${ }^{1}$ and M. Anayat ${ }^{2}$.

1. Mirpur University of Science and Technology (MUST), Mirpur-10250(AJK ), Pakistan.

2. International Islamic University Islamabad Pakistan.

\section{Manuscript Info}

\section{Manuscript History}

Received: 17 January 2017

Final Accepted: 07 February 2017

Published: March 2017

\section{Key words:-}

Mathematical modeling, Malaria disease, equilibrium, stability, basic reproduction number

\begin{abstract}
In this study, we present a compartmental model for the spread of malaria in a population where group of individuals were vaccinated. The purpose of this paper is to analyze the transmission dynamics of Malaria by using the compartmental model, including ordinary differential equations for human host and mosquito vector populations. A parallel system is obtained, which has two equilibriums: a diseasefree equilibrium and an endemic equilibrium. The stability of the equilibrium points is verified by the basic reproduction number $R_{0}$. Asymptotically stable solution is obtained only for disease-free equilibrium and results are presented graphically.
\end{abstract}

Copy Right, IJAR, 2017,. All rights reserved.

\section{Introduction:-}

Malaria is most common in Africa. The disease prevails in warmer climates and in areas where there is abundance of humidity and rain. According to latest WHO estimates released in December 2015, there were 214 million cases of malaria in 2015 and 438,000 deaths. At present five known species of plasmodium exists which are; $\mathrm{P}$ falciparum, $\mathrm{P}$ vivax, $\mathrm{P}$ ovale, $\mathrm{P}$ malariae and $\mathrm{P}$ knowlesi among them $\mathrm{P}$. falciparum is responsible for most fatal infection and is widespread in the tropics. Clinical symptoms of malaria include: high fever, muscle and joint aches, headache, vomiting, chills, sweating and anemia etc. Young children and travellers (who do not have developed immunity) are at high risk of malaria. In highly endemic areas people develop a degree of acquired immunity which is boosted-up by new infections. Immunity lasts for a certain period in the absence of new infection. The duration of infection depends on many factors such as degree of infection, method and time of treatment, resistivity of parasite to drugs and biology of host.

Mathematical modeling has been frequently used in epidemiology and several other fields. Epidemiological modeling is very helpful in identifying important model parameters and suggests improvements in models for future predictions.

We modified the model of NidhiNirwani, R. Khandelwal and V.H. Badshah by performing the stability analysis. The purpose of this study is better understanding the dynamics of malaria. 


\section{Description of Model:-}

Let $\mathrm{N}_{\mathrm{H}}$ and $\mathrm{N}_{\mathrm{V}}$ denote human and mosquito population respectively with total population at time $\mathrm{t}$. We assume that human and mosquito population has constant size with birth and death rates equal to $\mu_{\mathrm{H}}$ and $\mu_{\mathrm{V}}$.

The human population of size $\mathrm{N}_{\mathrm{H}}$ is composed of Susceptible $\mathrm{S}_{\mathrm{H}}$, Infective $\mathrm{I}_{\mathrm{H}}$ and Recovered $\mathrm{R}_{\mathrm{H}}$ whereas vector population consists of Susceptible $S_{V}$ and Infective $I_{V}$.

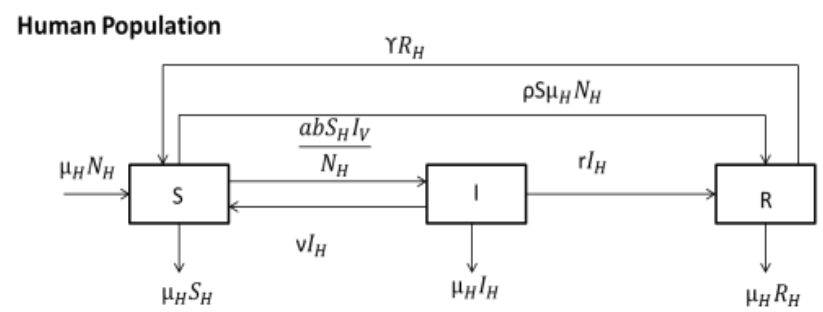

Vector Population

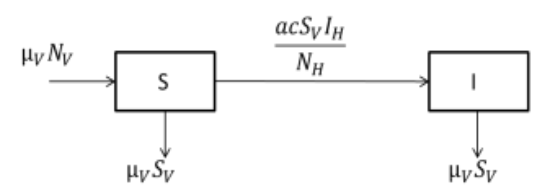

We say group of people $\rho, 0 \leq \rho \geq 1$ of newborns host population be vaccinated. Assume that vaccine is not perfect and let the effectiveness of vaccine is s, then $\mu_{H}(1-\rho s) N_{H}$ newborns remain susceptible, and $\mu_{H} \rho s N_{H}$ directly being removed to $R_{H}$. The governing equations are:

\section{Human population:-}

$\frac{d S_{H}}{d t}=\mu_{H}(1-\rho s) N_{H}-\frac{a b S_{H} I_{V}}{N_{H}}+v I_{H}+\gamma R_{H}-\mu_{H} S_{H}$

$\frac{d I_{H}}{d t}=\frac{a b S_{H} I_{V}}{N_{H}}-v I_{H}-r I_{H}-\mu_{H} I_{H}$

$\frac{d R_{H}}{d t}=\mu_{H} \rho s N_{H}+r I_{H}-\gamma R_{H}-\mu_{H} R_{H}$

\section{Vector Population:-}

$\frac{d S_{V}}{d t}=\mu_{V} N_{V}-\frac{a c S_{V} I_{H}}{N_{H}}-\mu_{V} S_{V}(2)$

$\frac{d I_{V}}{d t}=\frac{a c S_{V} I_{H}}{N_{H}}-\mu_{V} I_{V}$

The human population of size $\mathrm{N}_{H}$ is formed of susceptible $S_{H}$, infective $I_{H}$ and recovered $R_{H}$ where vector population is composed of $\mathrm{S}_{\mathrm{H}}$ and $\mathrm{I}_{\mathrm{H}}$.

Table 2.1:- Description of parameters of the model.

\begin{tabular}{|c|c|}
\hline Variables & Interpretation \\
\hline a & The average infection rate on man by single mosquito. \\
\hline b & The proportion of bites on man that produce an infection. \\
\hline c & The probability that a mosquito becomes infectious. \\
\hline r & The per capita rate of loss of immunity in human hosts. \\
\hline$v$ & The rate at which human hosts acquire immunity. \\
\hline & The rate of recovery of human hosts from the disease. \\
\hline
\end{tabular}

Using $S_{H}+I_{H}+R_{H}=N_{H}$ and $S_{V}+I_{V}=N_{V}$, eqs (1) and (2) become 
$\frac{d S_{H}}{d t}=\mu_{H}(1-\rho s) N_{H}-\frac{a b S_{H} I_{V}}{N_{H}}+v I_{H}+\Upsilon\left(N_{H}+S_{H}+I_{H}\right)-\mu_{H} S_{H}$

$\frac{d I_{H}}{d t}=\frac{a b S_{H} I_{V}}{N_{H}}-v I_{H}-r I_{H}-\mu_{H} I_{H}$

$\frac{d I_{V}}{d t}=\frac{a c I_{H}\left(N_{V}-I_{V}\right)}{N_{H}}-\mu_{V} I_{V}$

Writing the eq (2.3) in population proportion

$S_{h}=\frac{S_{H}}{N_{H}}, I_{h}=\frac{I_{H}}{N_{H}}$ and $I_{v}=\frac{I_{V}}{N_{V}}$,

$\frac{d S_{h}}{d t}=\mu_{H}(1-\rho s)-a b \phi S_{h} I_{v}+v I_{h}+\gamma-\gamma\left(S_{h}+I_{h}\right)-\mu_{H} S_{h}$

$\frac{d I_{h}}{d t}=a b \phi S_{h} I_{v}-v I_{h}-r I_{h}-\mu_{H} I_{h}$

$\frac{d I_{v}}{d t}=a c I_{h}\left(1-I_{v}\right)-\mu_{v} I_{v}$

Where $\phi=\frac{N_{V}}{N_{H}}$ is the ratio of host and vector population.

Further we rescale $\mathrm{t}$ by ac and let $x=S_{h}, y=I_{h}$ and $z=I_{v}$

$\frac{d x}{d t}=\mu(1-x)-\sigma x-\eta x z+k y+\varphi-\varphi y$

$\frac{d y}{d t}=\eta x z-(k+m) y$

$\frac{d z}{d t}=y(1-z)-\omega z$

Where

$\mu=\frac{\mu_{H}}{a c}, \pi=\rho s, \eta=\frac{b \phi}{c}, m=\frac{\mu_{H}+r}{a c}, \omega=\frac{\mu_{V}}{a c}, k=\frac{v}{a c}, \sigma=\frac{\mu_{H}+r}{a c}, \varphi=\frac{r}{a c}$

Euler Method:-

Values of parameters used were $\beta=0.000318$ and $\mu=0.0175$ and the initial conditions were $\mathrm{S}(0)=460, \mathrm{I}(0)$ $=12, \mathrm{R}(0)=0$

The Euler method

$$
\left\{\begin{array}{c}
\quad \begin{array}{c}
w_{0}=\alpha \\
w_{i+1}= \\
w_{i}+h f\left(t_{i}, w_{i}\right)
\end{array} \quad, i=0,1,2, \ldots \ldots \ldots, N-1
\end{array}\right.
$$

The time interval was [0 90] and $\mathrm{N}=10$ so

$\mathrm{h}=\frac{b-a}{N}$

$\mathrm{h}=\frac{90-0}{10}=9$

$t_{0}=0, t_{1}=9, t_{2}=18, t_{3}=27, \ldots \ldots \ldots . ., t_{10}=90$

$$
w_{0}=\left(\begin{array}{c}
S(0) \\
I(0) \\
R(0)
\end{array}\right)=\left(\begin{array}{c}
460 \\
12 \\
0
\end{array}\right)=\left(\begin{array}{l}
w_{1,0} \\
w_{2,0} \\
w_{3,0}
\end{array}\right)
$$

Iteration- $1 \quad$ for $\mathrm{i}=0$

$$
\begin{gathered}
w_{1}=w_{0}+h f\left(t_{0}, w_{0}\right) \\
w_{1}=\left(\begin{array}{l}
w_{1,0} \\
w_{2,0} \\
w_{3,0}
\end{array}\right)+h f\left(0, w_{0}\right) \\
w_{1}=\left(\begin{array}{c}
460 \\
12 \\
0
\end{array}\right)+9\left(\begin{array}{c}
-0.00038 \\
-0.20962 \\
0.21
\end{array}\right)
\end{gathered}
$$




\section{Steady State And Equilibrium Points:-}

The system (5) has a disease-free equilibrium point $\mathrm{E}_{0}\left(\frac{\mu(1-\pi)+\varphi}{\sigma}, 0,0\right)$ and an endemic equilibrium point $\mathrm{E}_{1}\left(\mathrm{x}_{\mathrm{e}}, \mathrm{y}_{\mathrm{e}}\right.$ , $\left.\mathrm{z}_{\mathrm{e}}\right)$, where

$x_{e}=\frac{(k+m)[\mu(1-\pi)+\varphi+(m+\varphi) \omega]}{\eta(m+\varphi)+\sigma(k+m)}$

$=\frac{\left(v+\mu_{H}+r\right)\left[a c\left\{\mu_{H}(1-\rho s)+\gamma\right\}+\left(\mu_{H}+r\right) \mu_{V}\right]}{a c\left[a b \phi\left(\mu_{H}+r+\gamma\right)+\left(\mu_{H}+\gamma\right)\left(v+\mu_{H}+r\right)\right]}$

$y_{e}=\frac{\eta[\mu(1-\pi)+\varphi]-\sigma(k+m) \omega}{\eta(m+\varphi)+\sigma(k+m)}$

$=\frac{a^{2} b c \phi\left[\mu_{H}(1-\rho s)+\Upsilon\right]-\left(\mu_{H}+\Upsilon\right)\left(v+\mu_{H}+r\right) \mu_{V}}{a c\left[a b \phi\left(\mu_{H}+r+\gamma\right)+\left(\mu_{H}+\Upsilon\right)\left(v+\mu_{H}+r\right)\right]}$

$z_{e}=\frac{\eta[\mu(1-\pi)+\varphi]-\sigma(k+m) \omega}{\eta[\mu(1-\pi)+\varphi+(m+\varphi) \omega]}$

$=\frac{a^{2} b c \phi\left[\mu_{H}(1-\rho s)+\gamma\right]-\left(\mu_{H}+\gamma\right)\left(v+\mu_{H}+r\right) \mu_{V}}{a b \phi\left[a c\left\{\mu_{H}(1-\rho s)+\gamma\right\}+\left(v+\mu_{H}+r\right) \mu_{V}\right]}$

This has been obtained by setting the time derivatives of the Eq (5) equal to zero. Here the basic reproduction number $\mathrm{R}_{0}$ is defined by

$$
R_{0}=\frac{\eta[\mu(1-\pi)+\varphi]}{\sigma(k+m) \omega}=\frac{a^{2} b c \phi\left[\mu_{H}(1-\rho s)+\Upsilon\right]}{\left(\mu_{H}+\Upsilon\right)\left(v+\mu_{H}+r\right) \mu_{V}}
$$

And an endemic equilibrium $\mathrm{E}_{1}\left(\mathrm{x}_{\mathrm{e}}, \mathrm{y}_{\mathrm{e}}, \mathrm{z}_{\mathrm{e}}\right)$ is stable when

$$
\begin{gathered}
R_{0}=\frac{\eta[\mu(1-\pi)+\varphi]}{\sigma(k+m) \omega}>0 \\
w_{1}=\left(\begin{array}{c}
459.99658 \\
10.11342 \\
1.89
\end{array}\right)
\end{gathered}
$$

\section{Asymptotic Behaviour Of The Model:-}

Theorem 1: If $\mathrm{R}_{0}<1$, then the disease-free equilibrium is locally $\mathrm{E}_{0}$ is locally stable and if $\mathrm{R}_{0}=1, \mathrm{E}_{0}$ is stable.

\section{Proof:}

To discuss the stability of the model, the governing dynamical system is

$F_{1}=\mu(1-\pi)-\sigma x-\eta x z+k y+\varphi-\varphi y$

$F_{2}=\eta x z-k y-m y$

$F_{3}=y(1-z)-\omega z$

The variation matrix of the above system is given by

$$
J=\left[\begin{array}{ccc}
-\sigma-\eta z & k-\varphi & -\eta x \\
\eta z & -k-m & \eta x \\
0 & 1-z & -y-\omega
\end{array}\right]
$$

For disease free equilibrium $\mathrm{E}_{0}\left(\frac{\mu(1-\pi)+\varphi}{\sigma}, 0,0\right)$ the variation matrix will be

$$
J\left(E_{0}\right)=\left[\begin{array}{ccc}
-\sigma & k-\varphi & \frac{-\eta[\mu(1-\pi)+\varphi]}{\sigma} \\
0 & -k-m & \frac{\eta[\mu(1-\pi)+\varphi]}{\sigma} \\
0 & 1 & -\omega
\end{array}\right]
$$

The characteristic equation of it will be

$(\sigma+\lambda)\left[\lambda^{2}+(k+m+\omega) \lambda+(k+m) \omega-\frac{\eta[\mu(1-\pi)+\varphi]}{\sigma}\right]=0(9)$

By the above equation at eigenvalues, one can easily see that disease-free equilibrium $\mathrm{E}_{0}$ is locally stable if 
i.e., $\mathrm{R}_{0}<1$

$$
(k+m) \omega-\frac{\eta[\mu(1-\pi)+\varphi]}{\sigma}>0
$$

Stability of disease-free equilibrium:-

The assumed values of all the parameters are given in the table below:

\begin{tabular}{|c|c|}
\hline Parameters & Values \\
\hline$\mu$ & 1.16 \\
\hline$\eta$ & 0.00492 \\
\hline$\pi$ & 0.009 \\
\hline$\sigma$ & 2.1724 \\
\hline$\phi$ & 0.9 \\
\hline $\mathrm{k}$ & 0.0026 \\
\hline$\omega$ & 1 \\
\hline $\mathrm{m}$ & 0.011 \\
\hline
\end{tabular}

With initial conditions $(x(0), y(0), z(0))=(0.5,0.5,0.4)$

After substituting the values in equation (4.4) we get;

$(2.1724+\lambda)\left(\lambda^{2}+1.0136 \lambda\right)+0.0089=0(10)$

Where,

$\lambda_{1}=-2.1724$

$\lambda_{2}=-0.0089$

$\lambda_{3}=-1.00475$

We see all Eigen values are real and negative, also $R_{0}=0.3413084471<1$, so the system is stable.

\section{Stability of disease-free equilibrium:-}

Taking equations (6) and (7); the variation matrix is given by

$$
J_{1}=\left[\begin{array}{cc}
-\sigma-\eta z & k-\varphi \\
\eta z & -k-m
\end{array}\right]
$$

For disease free equilibrium $\mathrm{E}_{0}\left(\frac{\mu(1-\pi)+\varphi}{\sigma}, 0,0\right)$ the variation matrix will be

$$
J_{1}=\left[\begin{array}{cc}
-\sigma & k-\varphi \\
0 & -k-m
\end{array}\right]
$$

And putting the values from the table of disease-free equilibrium, we will get the following linear system;

Now we take the graph of above equation through p-plane

$$
\begin{gathered}
\dot{x}=-2.1724 x-0.89747 y \\
\dot{y}=-0.0136 y
\end{gathered}
$$

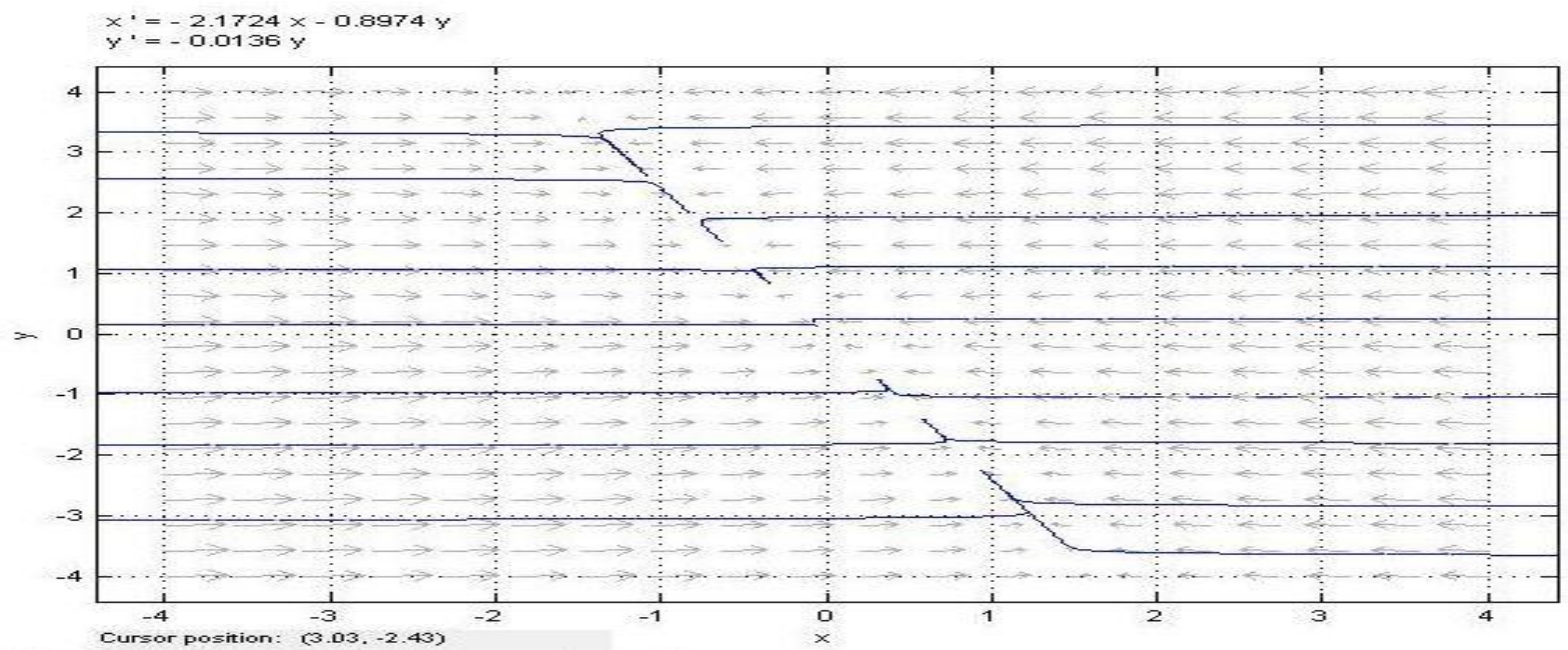


Now, taking equations (6) and (7); the variation matrix is given by

$$
J_{2}=\left[\begin{array}{cc}
-k-m & \eta x \\
1-z & -y-\omega
\end{array}\right]
$$

For disease free equilibrium $\mathrm{E}_{0}\left(\frac{\mu(1-\pi)+\varphi}{\sigma}, 0,0\right)$ the variation matrix will be

$$
J_{1}=\left[\begin{array}{cc}
-k-m & \frac{\eta[\mu(1-\pi)+\varphi]}{\sigma} \\
1 & -\omega
\end{array}\right]
$$

And putting the values from the table of disease-free equilibrium, we will get the following linear system;

$$
\begin{gathered}
\dot{y}=-0.0136 y+0.004047 z \\
\dot{z}=y-z
\end{gathered}
$$

Now we take the graph of above equation through p-plane

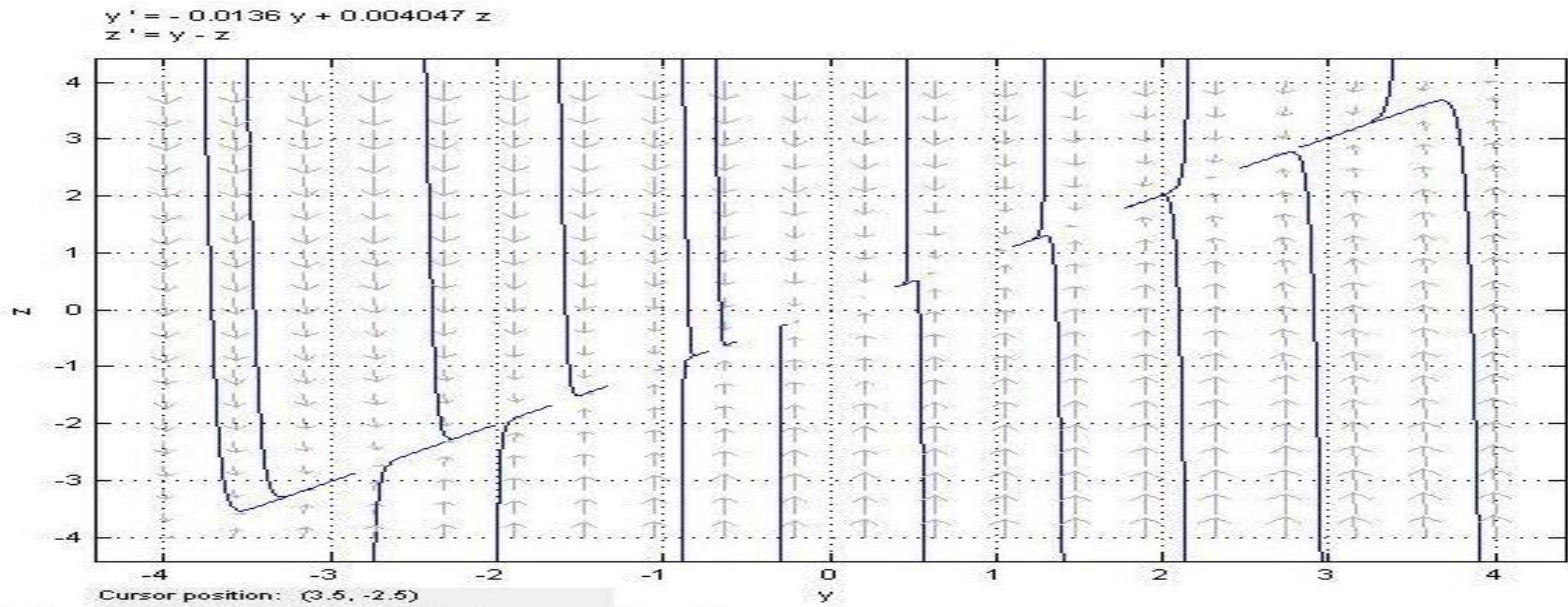

Now, taking equations (6) and (9); the variation matrix is given by

$$
J_{3}=\left[\begin{array}{cc}
-\sigma-\eta z & \eta x \\
0 & -y-\omega
\end{array}\right]
$$

For disease free equilibrium $\mathrm{E}_{0}\left(\frac{\mu(1-\pi)+\varphi}{\sigma}, 0,0\right)$ the variation matrix will be

$$
J_{1}=\left[\begin{array}{cc}
-\sigma & \frac{\eta[\mu(1-\pi)+\varphi]}{\sigma} \\
0 & -\omega
\end{array}\right]
$$

And putting the values from the table of disease-free equilibrium, we will get the following linear system;

$$
\begin{gathered}
\dot{x}=-2.1724 x+0.004047 z \\
\dot{z}=-z
\end{gathered}
$$

Now we take the graph of above equation through p-plane 


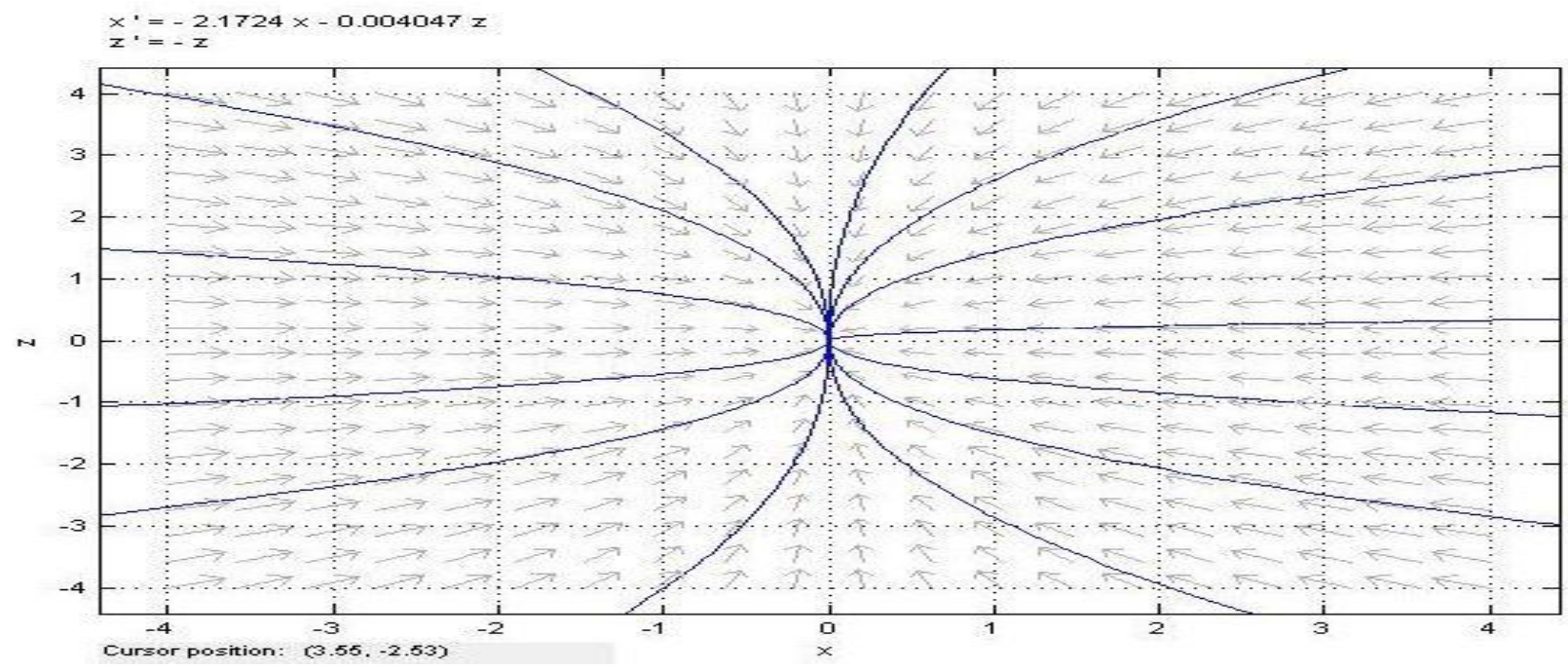

\section{Conclusion}

In this paper we present compartmental model for spread of malaria. An identical system is obtained which has two equilibrium points; a disease-free equilibrium and an endemic equilibrium. We discussed the stability of the diseasefree equilibrium point numerically by computing Eigen-values and by using basic reproduction number. Further we perform stability analysis and graphically verify our results. All the graph indicate that the system is stable.

\section{Future Work}

The model presented here is multi-dimensional. In future we can perform stability analysis for endemic-equilibrium. Furthermore we can establish these results by using any of the numerical methods. Also we can work on its sensitivity analysis and parameter estimation.

\section{References:-}

1. Anderson, R. M., \& May, R.M. (1991). Infectious Diseases of Humans: Dynamics and Control, Oxford University Press, Oxford.

2. [2] Aron, J.L. (1988). Mathematical modeling of immunity to malaria. Mathematical Biosciences, 90, 385396. http://dx.doi.org/10.1016/0025-5564(88)90076-4

3. [4] Hethcote, H. W. (1976). Qualitative analysis of communicable disease models. Mathematical Biosciences, 28, 335-356. http://dx.doi.org/10.1016/0025-5564(76)90132-2

4. [5] Ngwa, G. A., \&Shu, W.S. (2000). A mathematical model for endemic malaria with variable human and mosquito populations. Mathematics and Computer Modelling, 32, 747-763. http://dx.doi.org/10.1016/S08957177(00)00169-2

5. [6] Olumese, P. (2005). Epidemiology and surveillance: changing the global picture of malaria-myth or reality? ActaTropica, 95, 265-269. http://dx.doi.org/10.1016/j.actatropica.2005.06.006

6. [7] Sachs, J. D. (2000).A new global effort to control malaria. Science, 298, 122-124. http://dx.doi.org/10.1126/science. 1077900

7. [8] Tumwiine, J., Luboobi, L.S., Mugisha, J.Y. T. (2007). A mathematical model for the dynamics of malaria in a human host and mosquito vector with temporary immunity. Applied Mathematics and Computation, 189, 1953-1956. http://dx.doi.org/10.1016/j.amc.2006.12.084

8. [9] Tumwiine, J., Luboobi, L. S., \& Magical, J. Y. T. (2005).Modelling the effect of treatment and mosquitoes control on malaria transmission.International Journal of Management and Systems, 21, 107-124. 